INPLASY

PROTOCOL

To cite: Zhang et al. Less invasive versus conventional left ventricular assist device exchange: A protocol for systematic review and metaanalysis. Inplasy protocol

202180110. doi:

10.37766/inplasy2021.8.0110

Received: 29 August 2021

Published: 29 August 2021

Corresponding author:

Zhigang Liu

liuzgtedaich@163.com

Author Affiliation:

TEDA International

Cardiovascular Hospital.

Support: NSFTC.

Review Stage at time of this submission: Preliminary searches.

Conflicts of interest:

None declared.

\section{Less invasive versus conventional left ventricular assist device exchange: A protocol for systematic review and meta-analysis}

\author{
Zhang, B1; Guo, S2; Liu, Z33.
}

Review question / Objective: Can less invasive approach bring more benefits for patients requiring left ventricular assist device exchange, compared with the conventional approach? Participant or population: The population of interest will be patients, aged 18 and over, undergoing LVAD exchange because of device complications. Trials will be excluded if the study population is mainly composed of infants or children. Information sources: The electronic search will be conducted from PubMed, Cochrane Library, Web of Science, Scopus, and Embase databases until Aug 10, 2021, supplemented with manual screening for references in identified articles.

INPLASY registration number: This protocol was registered with the International Platform of Registered Systematic Review and Meta-Analysis Protocols (INPLASY) on 29 August 2021 and was last updated on 29 August 2021 (registration number INPLASY202180110).

\section{INTRODUCTION}

Review question / Objective: Can less invasive approach bring more benefits for patients requiring left ventricular assist device exchange, compared with the conventional approach?
Condition being studied: Heart failure, as an increasing global disease, is an advanced clinical stage of cardiovascular disease with high morbidity and mortality. Over 64 million people worldwide suffer from heart failure, which seriously affects 
their quality of life. Left ventricular assist device (LVAD) implantation has become a widespread and effective approach for advanced heart failure due to the paucity of donor hearts. However, adverse events still pose variable risks for patients although the device is constantly being optimized and improved. Pump thrombosis, as a significant and emergent adverse event, has become the main reason for device exchange. The potential mechanism is that abnormal activation of the coagulation system after exogenous device implantation result in the occurrence of thrombosis and even stroke. Besides, device malfunction and device-related infection can also lead to pump exchange. It has been reported that surgical pump exchange is associated with better effectiveness and prognosis for pump thrombosis compared with medical management. Therefore, some patients are hard to avoid pump exchange due to device complications during long-term LVAD support. The conventional approach refers to perform pump exchange via median sternotomy. Nowadays, some studies have reported axial-flow or centrifugal-flow pump exchange by less invasive techniques without full sternotomy. Typically, the old pump is removed and the new device is put in place by a subcostal incision. The rib-cross incision can be performed when the inflow site cannot be well exposed. Most current literature is limited to a small number of patients and inconsistent results. There is no consensus on whether less invasive techniques can bring more benefits for patients compared with the conventional technique. Here, our study will mainly discuss the comparison of less invasive and conventional techniques for patients requiring LVAD exchange.Left ventricular assist device (LVAD) implantation has become a widespread and effective approach for advanced heart failure. Some patients are hard to avoid pump exchange due to device complications during longterm LVAD support. Our study will mainly discuss the comparison of less invasive and conventional techniques for patients requiring LVAD exchange.

\section{METHODS}

Participant or population: The population of interest will be patients, aged 18 and over, undergoing LVAD exchange because of device complications. Trials will be excluded if the study population is mainly composed of infants or children.

Intervention: Trials will be included in which patients undergoing LVAD exchange by less invasive techniques. Less invasive techniques will be defined as exchanging pump without full sternotomy.

Comparator: Trials will be included in which patients undergoing LVAD exchange by the conventional technique. The conventional technique will be defined as exchanging pump via median full sternotomy.

Study designs to be included: Interventional and observational studies will be included. Case reports, review articles, editorials, and low quality conference abstracts will be excluded from this study.

Eligibility criteria: The population of interest will be patients, aged 18 and over, undergoing LVAD exchange because of device complications. Trials will be excluded if the study population is mainly composed of infants or children.

Information sources: The electronic search will be conducted from PubMed, Cochrane Library, Web of Science, Scopus, and Embase databases until Aug 10, 2021, supplemented with manual screening for references in identified articles.

Main outcome(s): The primary outcomes are in-hospital mortality and long-term mortality. The secondary outcomes are adverse events including neurological dysfunction, major infection, respiratory failure, major bleeding, renal dysfunction, and severe right heart failure. Adverse events will be assessed according to Interagency Registry of Mechanically Assisted Circulatory Support (INTERMACS) definitions. 
Additional outcome(s): Other peri- and postoperative outcomes will be also analyzed, including cardiopulmonary bypass (CPB) time, operation time, bloodproduct utilization, postoperative mechanical ventilation time, length of intensive care unit (ICU) stay, and length of hospital stay. Blood-product utilization will be defined as intraoperative blood-product transfusion.

Quality assessment / Risk of bias analysis: The quality of the included literature will be assessed according to the NewcastleOttawa Scale (NOS). The assessment scale is divided into three parts: selection (4 points), comparability ( 2 points), and outcome (3 points), including 7-9 points for low risk, 4-6 points for medium risk, and 0-3 points for high risk. Two reviewers (B.Z. and S.G.) will separately perform the quality assessment. The conflicts will be judged by consensus and discussion with a third researcher (Z.L.).

Strategy of data synthesis: Continuous data will be reported as mean and standard deviation (mean \pm SD). Dichotomous and continuous outcomes will be separately presented as odds ratios (OR) and standardized mean differences (SMD) with 95\% confidence intervals (Cl). A fixedeffects model will be used if no significant heterogeneity is detected; otherwise, a random-effects model will be used to pool the data. Potential publication bias will be evaluated by funnel plot and Egger's test. An absence of publication bias with Egger's test is considered when the Pvalue is $>0.05$. $I^{2}$ test will be used to estimate the heterogeneity. The heterogeneity will be considered significant if $\mathrm{I}^{2}$ is $>\mathbf{5 0 \%}$. Sensitivity analysis, subgroup analysis, and meta-regression will be used to explore the reason for considerable heterogeneity. Statistical significance will be considered if $P$-value is $<0.05$. Statistical analysis will be performed using Review Manager version 5.3 and Stata version 16. If meta-analysis is not appropriate to be performed, the results of all outcomes will be demonstrated narratively.
Subgroup analysis: Left ventricular assist device includes pulsatile-flow LVAD and continuous-flow LVAD. Pulsatile-flow LVADs mainly refer to first-generation LVADs, such as Heartmate XVE and Novacor. With the gradual advancement of equipment, continuous-flow LVADs have currently become the main devices because of their miniaturization and improved durability, including second- and third-generation pumps. Secondgeneration LVADs, like Heartmate II, pump blood through axial-flow technology and third-generation LVADs apply a centrifugalflow pattern with magnetically levitated forces, including Heartware HVAD and Heartmate 3. Subgroup analysis will be completed according to the type of left ventricular assist device if enough data can be available. In addition, we will also perform stratified analysis based on study quality, study design, and patients' characteristics to detect the reason for potential data inconsistency.

Sensitivity analysis: If needed, sensitivity analysis will be conducted to evaluate the robustness of the cumulative evidence and detect the potential sources of heterogeneity by excluding low quality articles or studies with different characteristics from the others.

Country(ies) involved: China.

Keywords: continuous-flow left ventricular assist device; minimally invasive techniques; the conventional technique; heart failure.

Contributions of each author:

Author 1 - Bufan Zhang.

Author 2 - Shaohua Guo.

Author 3 - Zhigang Liu. 